\title{
Lower Blepharoplasty Review, Transconjunctival vs. Transcutaneous Approach
}

\author{
Alberto Rancati*, Patricio Jacovella, Andrea Edoardo Zampieri, Julio Dorr, \\ Mariana Daniele, Santiago Liedtke, Agustin Rancati
}

Plastic Surgery Division, University of Buenos Aires (UBA), Buenos Aires, Argentina

Email: ${ }^{*}$ rancati@gmail.com

Received 20 October 2014; revised 20 November 2014; accepted 30 November 2014

Academic Editor: Guillermo Vazquez, FILACP, Argentina

Copyright (C) 2015 by authors and Scientific Research Publishing Inc.

This work is licensed under the Creative Commons Attribution International License (CC BY). http://creativecommons.org/licenses/by/4.0/

c) (i) Open Access

\begin{abstract}
Currently in lower blepharoplasty, the transconjunctival approach indication has been limited to young patients without skin excess and prominence of bags; in our practice this access has become the preferred technique in most of our cases because it is simpler, faster, prevents bad scarring, produces less orbicularis muscle trauma, and decreases postoperative edema and possible retractions (ectropion) in patients with decreased lower palpebral tone. Objective: To identify important concepts when deciding this approach. Material and Methods: Retrospective review of 177 patients who underwent lower blepharoplasty by a single surgical group. Regarding techniques, the transconjunctival approach was selected in $42 \%$ of patients while the transcutaneous technique was preferred in $58 \%$. Results: A lower rate of complications was observed by the transconjunctival approach, with greater patient satisfaction. Conclusions: In our experience, due to its simplicity and less traumatic effect on the patient, the transconjunctival approach is an ideal technique, except in cases where there is lower eyelid weakness and surgical resolution is needed.
\end{abstract}

\section{Keywords}

Transconjunctival Approach, Transcutaneous Approach, Blepharoplasty

\section{Introduction}

Lower blepharoplasty by the transconjunctival approach is not a substitute for the transcutaneous approach;

*Corresponding author.

How to cite this paper: Rancati, A., Jacovella, P., Zampieri, A.E., Dorr, J., Daniele, M., Liedtke, S. and Rancati, A. (2015) Lower Blepharoplasty Review, Transconjunctival vs. Transcutaneous Approach. Modern Plastic Surgery, 5, 1-8.

http://dx.doi.org/10.4236/mps.2015.51001 
however, when the fundamental problem is the prominence of fat bags, with no skin excess, or when for some reason the surgeon wants to avoid scaring on the eyelid, this approach in our experience should be considered in young patients. The objective of this communication is to point out some important tips when deciding this approach. Currently in our practice, transconjunctival access has become the preferred technique in the majority of cases. Besides the advantage of avoiding the scar, we consider this approach simpler and much faster than the conventional transcutaneous approach, avoiding incision through the orbicularis muscle, which preserves its innervation, diminishes postoperative edema, and retractions [1]. This approach also reduces the postoperative risk of lower eyelid retraction, especially indicated in patients who have a slight decrease in horizontal eyelid tone. In such cases of tarsal laxity, the technique may be combined with a tarsal strip to improve tension and avoid scleral show.

Preoperative evaluation includes different parameters and characteristics, such as evaluation of the skin and fat bags. The magnitude of the lipectomy should be planned preoperatively, to avoid excessive periorbital fat removal due to excessive resection of the fat bags.

Following the primary conjunctival incision, the medial and central fat pads are usually observed first. The lateral fat pad is covered with more septa than the central fat pad, and the fat may not spring forward as easily. After the superficial portion of the lateral fat pad has been excised, the posterior fat comes forward more freely. Moreover, it is important to start the resection by the medial or center fat bags, because it becomes less apparent and more difficult to resect with the possibility of being under corrected, thus, requiring a second surgery.

For evaluating the horizontal lower eyelid tone the "snap test" should be performed, in which a finger is used to push down the lower eyelid of the patient, observing the position taken by the ciliary margin over the eye during the release, without any flicker. If the eyelid returns immediately to a normal position, the tone is good. If the eyelid slowly returns to its original position, its tone is considered to be moderately poor, and it may not require horizontal lid handling, but any skin resection is contraindicated; these cases are considered ideal for the transconjunctival approach. If the eyelid returns to its original position with blinking movements, its tone is poor, and it will be essential to jointly treat the blepharoplasty with a tarsal strip. The dynamics of blinking and eyelid closure should also be noted.

Since blepharoplasty is a cosmetic procedure, we always take a preoperative photograph to explain the technique to the patient, and a postoperative photograph. Thus, it is essential to always take the same photographic views in each case. In our experience, the most useful photographic views are the following: 1) the frontal face view, which includes from ear to ear, and neck to the entire hair, but without unnecessary space exploration; 2) the oculoplasty approach which includes the entire face, nose, and auricular region; and 3) the right and left side outlets, covering the lateral orbital rim to the nasal bridge, attempting to show the superior sulcus, redundant skin in both eyelids, and fat protruding through the septum.

Digital photographs are useful because they allow for immediate analysis, comparing the results in each shot, in both pre- and post evaluations, to demonstrate to the patient the advantages of the surgery and the results that could be achieved. Before surgery, it is essential to know exactly which are the patients' expectations from the procedure, and clearly inform them about the expected results. After cosmetic surgery, patients often forget about their previous physical aspect, feeling that the results are poorer than they expected. Today it is common to have a computer in the office, and digital cameras are accessible, making both, the photographic system and duplication of material, simple.

\subsection{Anatomical Considerations (Figure 1)}

As noted, the transconjunctival approach is easier than the transcutaneous technique and takes less time, however, it requires precise anatomical knowledge of the internal region because it can cause iatrogenic dry eye. The main risk points are handling of the inferior oblique muscle, orbital septum and difficult bleeding control.

From the surgical point of view, it is important to remember that similar to upper eyelid surgery, as we operate near the tarsus, when pulling with retractors, structures are so fused that it may be difficult to recognize them, and while we part the tarsus to the orbital rim, these structures grow apart by the preaponeurotic fat package.

The capsulopalpebral fascia originates from a fibrous connection attached to the inferior rectus muscle insertion tendon and splits into 2 portions as it reaches the inferior oblique muscle merging partially and surrounding the fibrous capsule. The 2 branches meet to form the Lockwood suspensory ligament, which later ascend to the eyelid margin and attaches to the tarsus. 


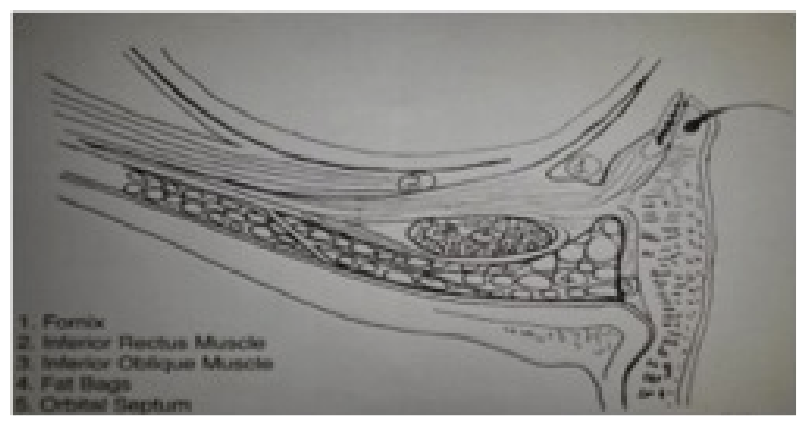

Figure 1. Ocular anatomy.

The inferior oblique muscle originates behind the posterior lacrimal crest, and as it reaches the globe, it separates the central and medial fat compartments. It is important for surgeons to learn to identify this structure which can be damaged during transconjunctival lipectomy.

Meticulous hemostasis is critical during the entire procedure. The blood vessels associated with each fat compartment must be cauterized.

Since the orbital septum is fixed to the inferior orbital rim, any adhesion between the cut edge of the septum and a movable portion of the eyelid will result in lower lid displacement. However, this occurs much less frequently than in transcutaneous surgery.

\subsection{Team}

In the last 2 years, we have verified the usefulness of the $\mathrm{CO}_{2}$ laser in this surgery, but given that most surgeons do not have access to this expensive device, we will discuss the procedure with conventional techniques, mentioning variations regarding the laser, such as the need of bipolar cautery, the use of special eye protection, and other medical devices.

It is essential to note that the procedure characteristics should be recorded and discussed with each patient before surgery. One of the frequent mistakes due to lack of experience, is to improvise according to what is found during the surgery, which leads to results that were not expected, and therefore, to inquiries and complaints from the patients.

\subsection{Surgical Technique}

The surgery involves various stages: preparation, anesthesia, incision, traction and corneal protection, exposure of fat packages, lipectomy, lipectomy control, and end of surgery.

\subsection{Preparation}

Fasting for a minimum of 7 hours is recommended although this procedure only requires local anesthesia. In most cases, the anesthesiologist will administer intravenous sedation, which despite increasing costs, provides, from our point of view, the best conditions of comfort and cooperation from the patient.

Especially when a $\mathrm{CO}_{2}$ laser device is available, surgery may be performed in the office, and also with a basic sedation infrastructure. Before starting the infiltration, an eye shield should be placed, as laser surgery must meet the conditions of protection for each team member.

\subsection{Anesthesia}

Adequate infiltration of a local anesthetic is critical for patient comfort and hemostasis. To reduce the sensitivity of the conjunctival mucosa, it is advisable to applying tetracaine drops, followed by placement of a cotton ball with $10 \%$ lidocaine (in the presentation used by dentists) over the area. With these guidelines, deep topical anesthesia can be obtained, but if applied without prior preparation with ophthalmic tetracaine, the patient may feel severe burning.

Lidocaine with epinephrine 2\% mixed with bupivicaine is infiltrated in the subconjunctiva using a 27 - 30 gauge needle, approximately $2 \mathrm{~cm}$ along the lower lid between the tarsus and fornix. 


\subsection{Incision}

Although there are established techniques for the transconjunctival fornix approach [2]-[5], in our experience, performing a laser incision directly near the edge of the tarsus is more anatomically favorable. Otherwise, we recommend a very gentle incision with monopolar electrocautery to decrease bleeding. The lower eyelid is everted; 2 cc of lidocaine with epinephrine 5\% are injected; and 15 minutes later, the incision is made between the edge of the tarsus and conjunctival fornix. No special protection is required for electrocautery incision with a monopolar needle tip with coagulation energy of 20 (Valleylab Co.) (Figure 2).

\subsection{Traction and Corneal Protection}

When the incision is performed and after cauterizing bleeding vessels, it is helpful to pull on the conjunctival flap with a 6-0 nylon, and to cover the cornea with it. By using retractors, good exposure of the surgical field is achieved, and with a bit of pressure on the upper globe orbital septum, the yellow orbital fat bulges into the field (Figure 3 and Figure 4).

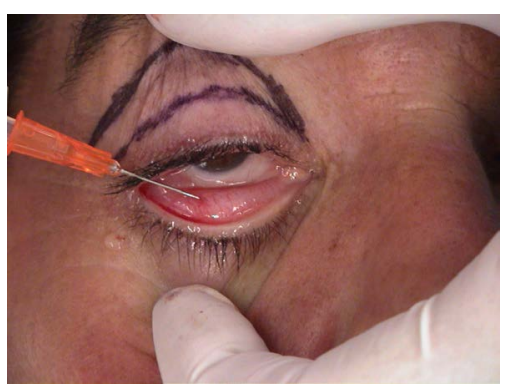

(a)

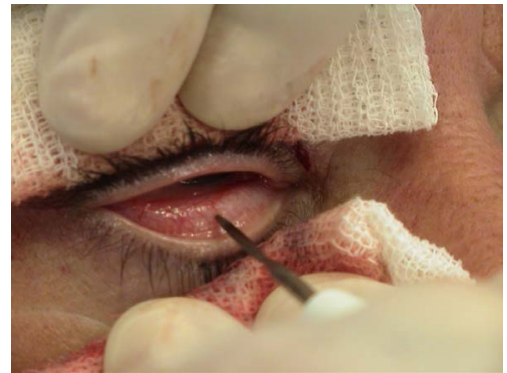

(b)

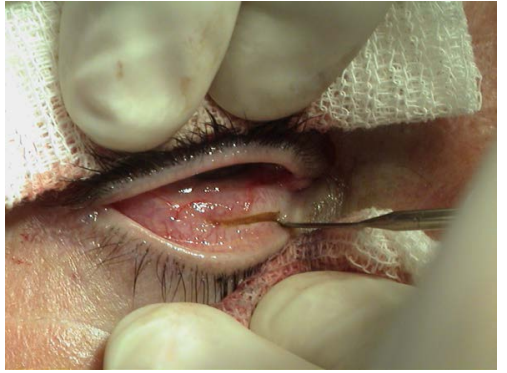

(c)

Figure 2. (a) lidocaine injection; (b) conjunctiva exposure; and (c) electrocautery incision.

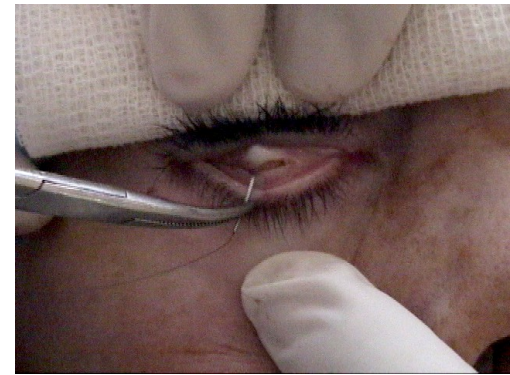

(a)

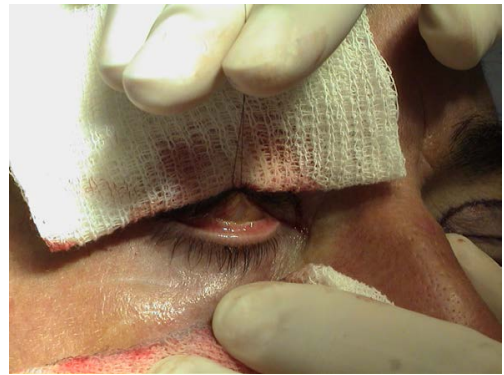

(b)

Figure 3. (a) After making the incision, the superior conjunctival flap is retracted to cover the cornea with 6-0 nylon. (b) The septum is exposed.

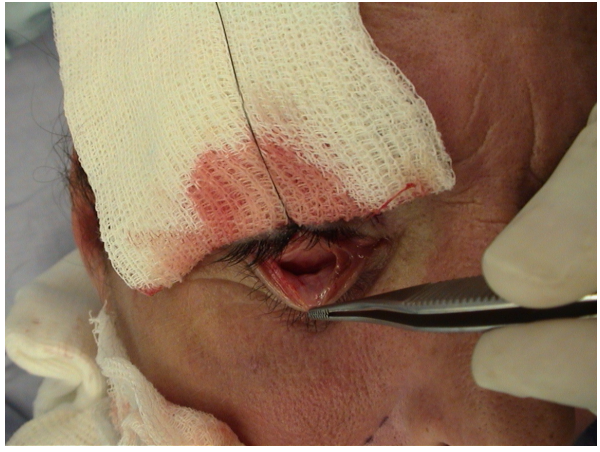

Figure 4. Traction is maintained with the nylon, and then the dissection of the septum can be continued. 


\subsection{Exposure of Fat Bags}

In the event that removal of the bag is required, it should be handled first, resecting the medial center before it becomes less apparent and difficult to address.

At the site where the fat protrudes through the septum, it must be opened by blunt dissection. This exagerates the protrusion while adding pressure in the eye ball (Figure 5). During this dissection, we recognize the inferior oblique muscle located between the medial and nasal bags. Although finding the oblique muscle is not necessary in all patients, we recommend identifying it at least in the first cases when the lipectomy is performed, to become familiar with its exact location and appearance at surgery (Figure 6).

\subsection{Lipectomy}

The lipectomy procedure has some variations, depending on the instruments with which the resection is made. In the conventional technique, it is recommended that once the fat is exposed, it should be clamped with a hemostat and cut afterwards, making hemostasis with bipolar cautery, preferably before releasing the clamp, since the fat will retract into the orbit, making it difficult to locate in the event of persistent bleeding vessels. Meticulous attention to hemostasis in this area should be carried out prior to closure.

Using the cutting mode, the radiosurgery equipment generally achieves coagulation with good control of small vessels, though these vessels are often over $1 \mathrm{~mm}$ in diameter; thus, the use of the cutting mode will depend on the appearance of the fat, which can vary from a healthy loose appearance without fibrosis or chronic inflammatory reaction to relatively fixed fibrous tissues with chronic inflammation (as in the case of dysthyroid orbitopathy).

When working with a monopolar energy electrocautery, cutting can be performed directly without clamping, achieving higher vessel coagulation.

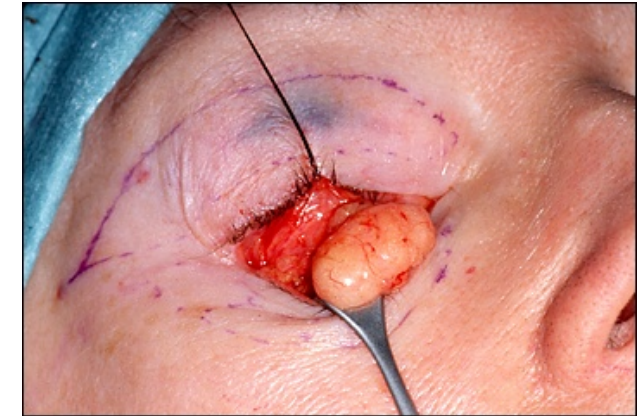

(a)

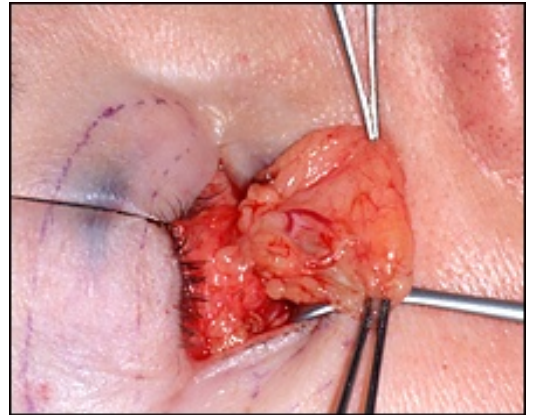

(b)

Figure 5. The lower conjunctival Desmarres retractor is used to expose the septum and underlying fat, which becomes more apparent by pressing the eyeball into the orbit.

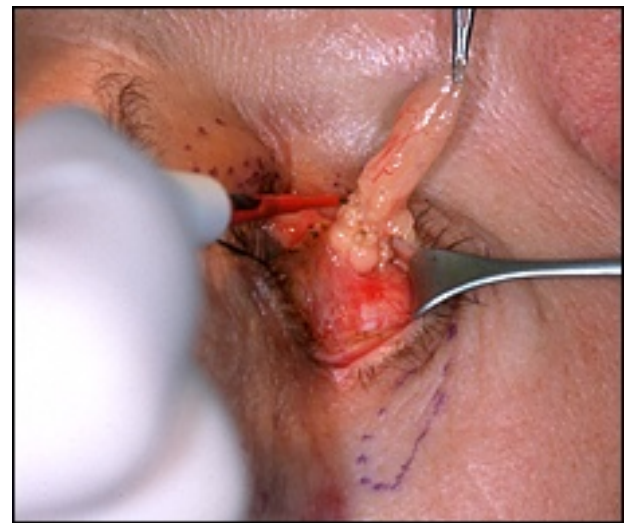

(a)

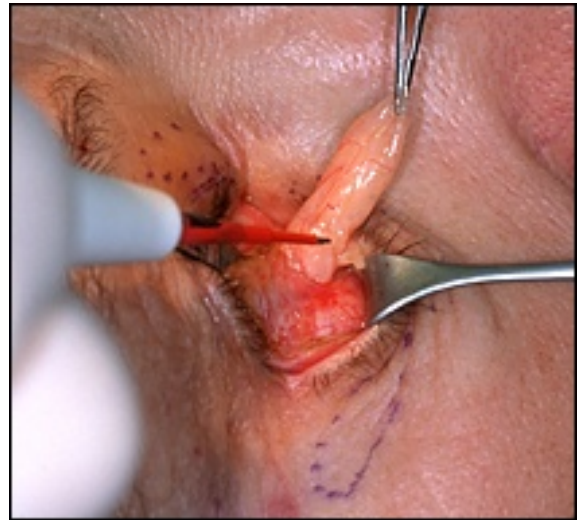

(b)

Figure 6. The septum is opened by pulling the exposed fatty bags, which are smoothed for resection. 


\subsection{Lipectomy Control}

After fat removal, the surgeon pulls down the lower lid margin upward to realign the tissue planes. With the lid stretched out, gentle pressure should be applied on the globe revealing any residual fat bulges. If necessary, further fat may be excised (Figure 7 and Figure 8) [6]-[10].

An advantage of using the transconjunctival approach is that stitches are not required. The conjunctiva is relocated face to face at the site of the resection, healing smoothly, and thus avoiding the risk of corneal damage.

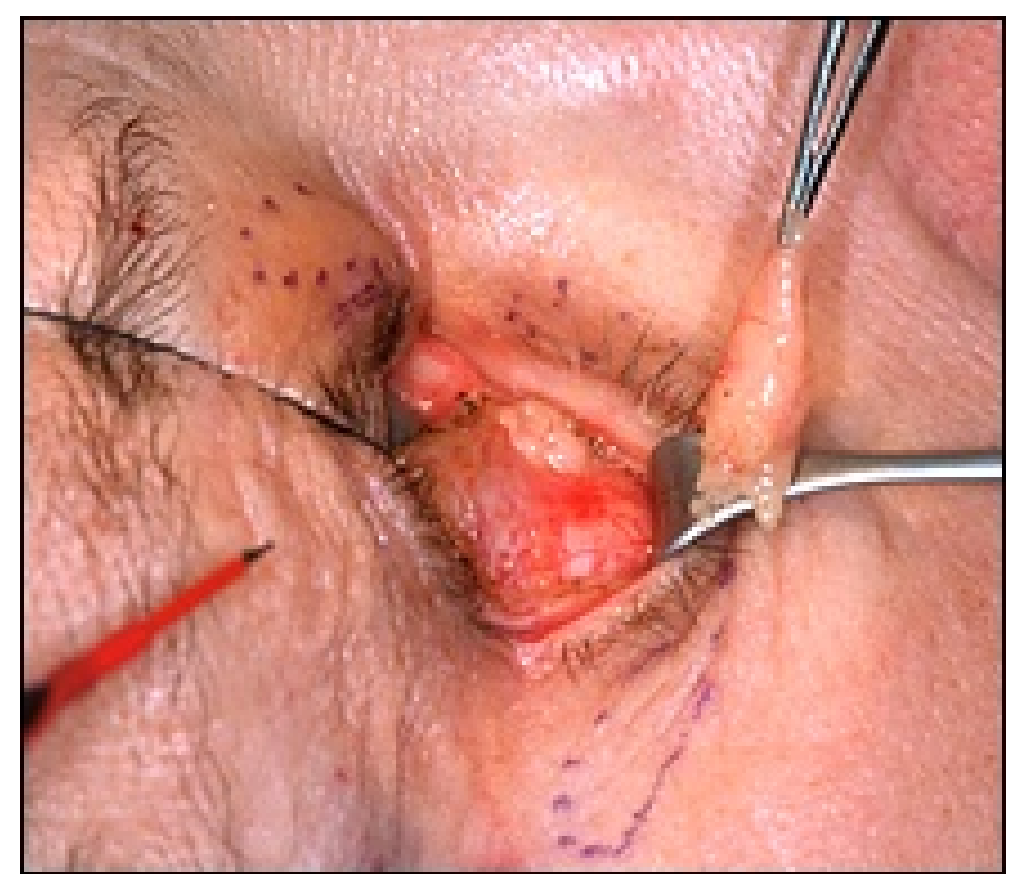

Figure 7. After fatty bag resection.

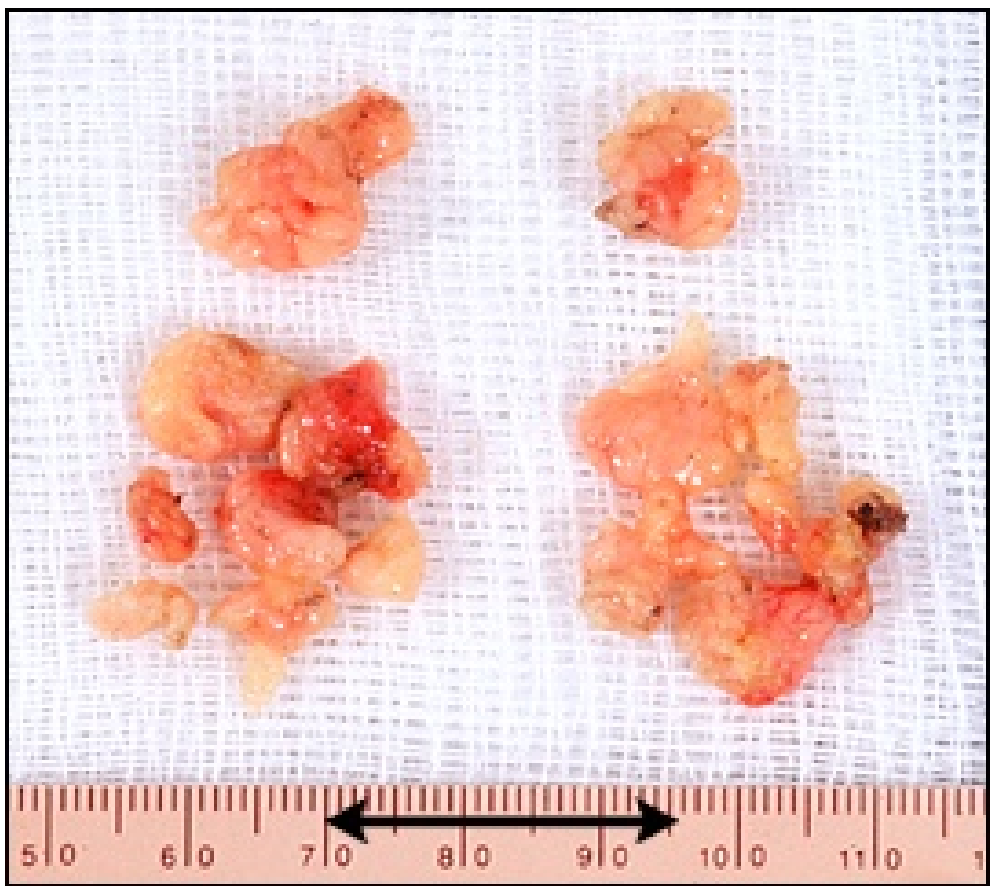

Figure 8. Resection of fat bags in both eyes. 


\subsection{End of Surgery}

It is useful to place a cold patch over the orbicular region at the intervention end. The patient is discharged, with oral anti-inflammatory, usually non-steroidal ointment twice a day and 2 cold packs mainly for the first 2 days after surgery.

\section{Materials and Methods}

This is a retrospective review of 177 patients who underwent lower blepharoplasty by a single surgical group. Seventy-five patients who underwent the transconjunctival approach (primary surgery, 67; secondary surgery, 8) and 102 patients who underwent the transcutaneous approach (primary surgery, 89; secondary surgery, 13) were followed up for 24 months (Table 1).

Patients were advised to use cold packs after the surgery for the first 2 days, no work for 3 - 4 days, and no physical activity for 10 days.

\section{Results}

Postoperative retro-orbital hemorrhage is the most feared complication following lower blepharoplasty. It can be accompanied with pain and decreased visual acuity with pupillary defect. In the transcutaneous approach, edema of the orbicularis muscle due to hemorrhage can affect visual acuity; with the transconjunctival approach, we avoid this complication, as well as cicatricial lower eyelid retraction with lagophthalmos and ectropion, and eyelid asymmetry, which are common complications of the classic transcutaneous approach. Some cases may require a second surgery correction because of insufficient removal of fat bags. Other complications include infection, scleral show, conjunctivitis, corneal ulcer, diplopia, amaurosis, and damage of both the inferior oblique muscle and the orbicularis oculi muscle [11] [12].

Table 2 shows the complications reported in patients who underwent the transconjunctival approach versus the transcutaneous approach. In our experience, among the patients in the transconjunctival group, $2.7 \%, 1 \%$, and $1 \%$ of patients presented insufficient lipectomy, bleeding, and corneal ulcer, respectively, and no infection, scleral show, conjunctivitis, or decreased visual acuity were reported. Among the patients who underwent the transcutaneous approach, $2 \%$, presented infection, $2 \%$, scleral show, $1 \%$, insufficient skin resection, $1 \%$, bleeding, and $1 \%$,corneal ulcer, respectively.

The patients' average period before returning back to work with was 10 days, regardless the approach.

The facial appearance of patients who underwent the transconjunctival approach did not reveal any surgery intervention.

Table 1. Statistics of primary surgery and secondary surgery.

\begin{tabular}{cccc}
\hline Patients & Primary surgery & Secondary surgery & Total $=177$ \\
\hline Transconjunctival & 67 & 8 & 75 \\
Trancutaneous & 89 & 13 & 102 \\
\hline
\end{tabular}

Table 2. Complications in the transconjunctival approach vs. transcutaneous approach.

\begin{tabular}{|c|c|c|}
\hline & Transconjunctival approach & Transcutaneous approach \\
\hline Insufficient lipectomy & $2.7 \%$ & $0 \%$ \\
\hline Bleeding & $1 \%$ & $1 \%$ \\
\hline Corneal ulcer & $1 \%$ & $1 \%$ \\
\hline Infections & $0 \%$ & $2 \%$ \\
\hline Scleral shows & $0 \%$ & $2 \%$ \\
\hline Insufficient skin resection & - & $1 \%$ \\
\hline
\end{tabular}




\section{Discussion}

The transconjunctival approach requires less surgery time than the transcutaneous approach, it guaranties less trauma on anatomical structures (surgeons must understand the complex interactions of all the structures comprising the eyelid, orbit, eye muscles), and allows for direct access to the surgery field. The transconjunctival approach also avoids suturing and cutaneous skin scaring with risk of retraction and ectropion. Furthermore, the post-operative recovery is faster with the transconjunctival approach. In our experience, the percentage of complications was lower and patient satisfaction was higher in the transconjunctival group.

Average time for transconjuntival approach was 45 minutes, vs. 60 minutes for transcutaneous.

\section{Conclusions}

In our experience, transconjunctival blepharoplasty is the best choice for lower blepharoplasty, when the herniated fat bags is the main problem and when skin excision is not necessary or we want to avoid it, since this technique allows us to associate with others as a tarsal strip in those patients with increased laxity.

Furthermore, this approach has the advantage of good mucosal scarring, minimal risk of tissue retraction and for our group has become the choice for patients with lower palpebral lipoptosis and good skin quality.

The complication rate is not higher than with the transcutaneous approach, and postoperative recovery time is reduced with greater comfort and less swelling.

\section{Declaration}

The authors declare no conflict of interest.

The protocol for the research project has been approved by a suitably constituted Ethics Committee of the institution.

\section{References}

[1] Hidalgo, D.A. (2011) An Integrated Approach to Lower Blepharoplasty. Plastic and Reconstructive Surgery, 127, 386395. http://dx.doi.org/10.1097/PRS.0b013e3181f95c66

[2] Collar, R.M., Lyford-Pike, S. and Byrne P. (2013) Algorithmic Approach to Lower Lid Blepharoplasty. Facial Plastic Surgery, 29, 32-39. http://dx.doi.org/10.1055/s-0033-1333836

[3] Fedok, F.G. and Perkins, S.W. (1996) Transconjunctival Blepharoplasty. Facial Plastic Surgery, 12, 185-195. http://dx.doi.org/10.1055/s-0028-1082409

[4] Weinberg, D.A. and Baylis, H.I. (1995) Transconjunctival Lower Eyelid Blepharoplasty. Dermatologic Surgery, 21, 407-410. http://dx.doi.org/10.1111/j.1524-4725.1995.tb00206.x

[5] Silkiss, R.Z. and Carroll, R.P. (1992) Transconjunctival Surgery. Ophthalmic Surgery, 23, 288-291.

[6] Goldberg, R.A., Edelstein, C. and Shorr, N. (1999) Fat Repositioning in Lower Blepharoplasty to Maintain Infraorbital Rim Contour. Facial Plastic Surgery, 15, 225-229. http://dx.doi.org/10.1055/s-2008-1064322

[7] Youn, S., Shin, J.I., Kim, J.T. and Kim, Y.H. (2013) Transconjunctival Subperiosteal Fat Reposition for Tear Trough Deformity: Pedicled Fat Redraping Versus Septal Reset. Annals of Plastic Surgery, 73, 479-484.

[8] Yoo, D.B., Peng, G.L. and Massry, G.G. (2013) Transconjunctival Lower Blepharoplasty with Fat Repositioning: A Retrospective Comparison of Transposing Fat to the Subperiosteal vs. Supraperiosteal Planes. JAMA Facial Plastic Surgery, 15, 176-181. http://dx.doi.org/10.1001/jamafacial.2013.749

[9] Couch, S.M., Buchanan, A.G. and Holds, J.B. (2011) Orbicularis Muscle Position during Lower Blepharoplasty with Fat Repositioning. JAMA Facial Plastic Surgery, 13, 387-391. http://dx.doi.org/10.1001/archfacial.2011.42

[10] Mohadjer, Y. and Holds, J.B. (2006) Cosmetic Lower Eyelid Blepharoplasty with Fat Repositioning via Intra-SOOF Dissection: Surgical Technique and Initial Outcomes. Ophthalmic Plastic \& Reconstructive Surgery, 22, 409-413. http://dx.doi.org/10.1097/01.iop.0000243607.36661.be

[11] Ghabrial, R., Lisman, R.D., Kane, M.A., Milite, J. and Richards, R. (1998) Diplopia Following Transconjunctival Blepharoplasty. Plastic and Reconstructive Surgery, 102, 1219-1225. http://dx.doi.org/10.1097/00006534-199809020-00049

[12] Yachouh, J., Arnaud, D., Psomas, C., Arnaud, S. and Goudot, P. (2006) Amaurosis after Lower Eyelid Laser Blepharoplasty. Ophthalmic Plastic \& Reconstructive Surgery, 22, 214-215.

http://dx.doi.org/10.1097/01.iop.0000214531.58587.57 
Scientific Research Publishing (SCIRP) is one of the largest Open Access journal publishers. It is currently publishing more than 200 open access, online, peer-reviewed journals covering a wide range of academic disciplines. SCIRP serves the worldwide academic communities and contributes to the progress and application of science with its publication.

Other selected journals from SCIRP are listed as below. Submit your manuscript to us via either submit@scirp.org or Online Submission Portal.
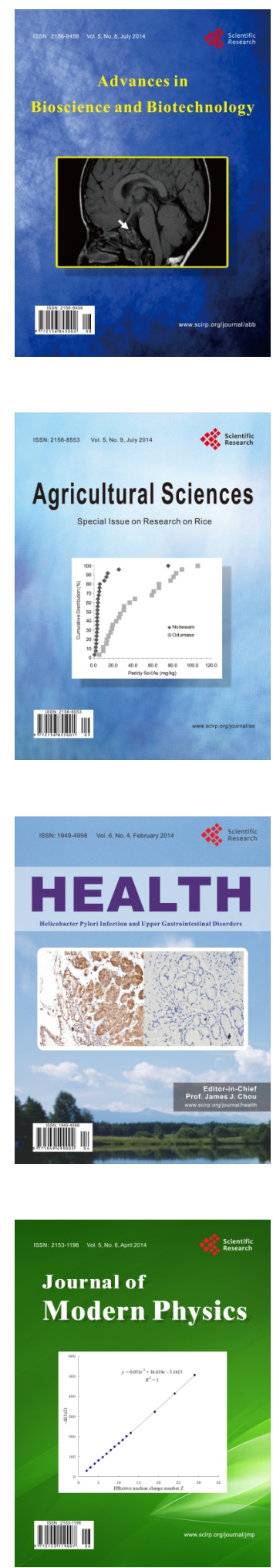
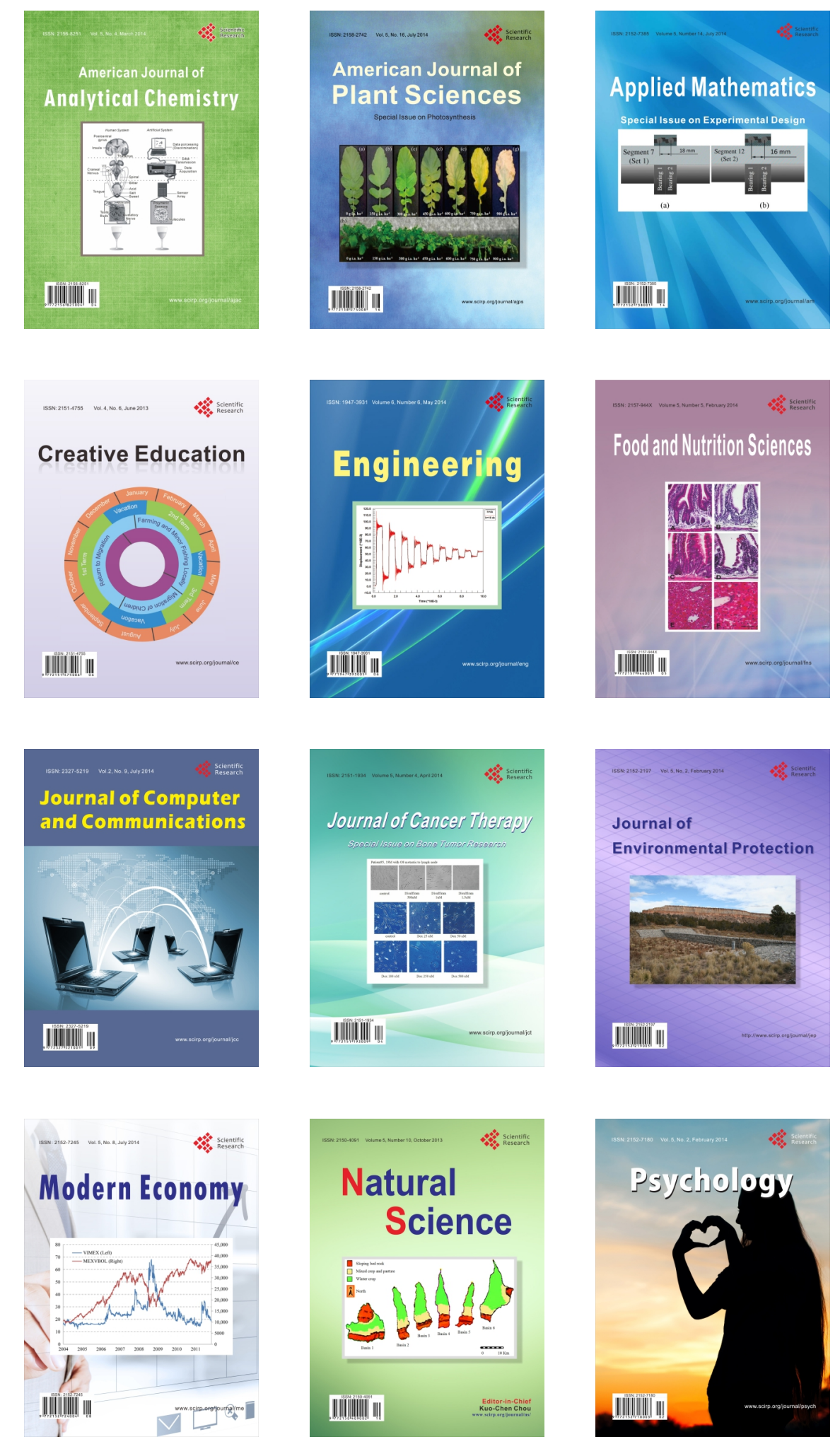